\title{
Rescue of Inherited Traits for Lethal Respiratory Diseases by Prenatal Gene Editing: More than One Single Gene and One
}

\section{Vector}

\author{
Di Gioia S* and Conese M \\ Department of Medical and Surgical Sciences, University of Foggia, Italy
}

*Corresponding author: Sante Di Gioia, Assistant Professor, Department of Medical and

\begin{tabular}{|c|}
\hline Commentary \\
Volume 4 Issue 1 \\
Received Date: July 03, 2019 \\
Published Date: July 31, 2019 \\
\hline
\end{tabular}

Surgical Sciences, University of Foggia, Italy, Tel: 3346929379; Email: sante.digioia@unifg.it

\section{Commentary}

A paper recently published in Science Translational Medicine [1] reports for the first time a proof of concept study on the potential application of in utero gene editing to face monogenic lung diseases that are lethal at birth. The authors, belonging to a team of researchers from the Children's Hospital of Philadelphia and Penn Medicine, focused their attention on a particular type of congenital lung disease characterised by mutations in an essential protein for lung function: the surfactant protein (SP). As compared to surfactant deficiency in premature infants, the inherited surfactant protein (SP) syndromes are more problematic in terms of therapeutic approach. Indeed, the respiratory failure secondary to SP syndrome cannot be treated with exogenous surfactant and other standard therapies. Indeed, treatment options for these neonatal patients are very limited, with the only options being palliative care or pediatric lung transplant. Therefore, an in utero gene editing could offer an alternative approach for the treatment of such a genetic disorder whose effects are devastating so early in development that gene therapy even in a new born would be too late.

The Philadelphia team has edited mouse genes by injecting CRISPR/Cas9 into the amniotic fluid of pregnant mice. CRISPR/Cas9 (clustered regularly interspaced short palindromic repeats/CRISPR associated protein 9) has managed, in the last 10 years, to become one of the main world scientific discovery since the implementation of polymerase chain reaction. The ability of this machinery to modify the function of different genes within living organisms has changed the pace of numerous biomedical research branches especially those concentrated on genetic driven pathologies [2]. CRISPR uses RNA to guide precise cuts in the genome, as follow:

1. A guide sequence of RNA (gRNA) is engineered to target a specific region of DNA.

2. The gRNA, linked to a Cas9 cleaving enzyme, finds the right spot on the genome then unzips the double helix and snips the gene.

3. Before the DNA is repaired, small amounts of engineered DNA can be inserted to alter the target gene.

It must be said that, despite being effectively useful in genome modification and functional regulation in biological systems, in vivo CRISPR/Cas9 application is still challenging because of the difficulties in reaching the precise target cells. Interestingly, the results obtained by these researchers are in some ways exciting as they showed specific targeting to just the affected organ, and even to specific cells within the lung. This specific targeting of lung tissues is in partly due to the combination by breathing movements and the direct contact with the developing airways and amniotic fluid suggesting that CRISPR/Cas9administered via the intraamniotic route could reach the lung epithelium. For the first time, it has been demonstrated that a timed in utero delivery of an adenoviral (Ad) vector carrying CRISPRCas 9 is able to reach lungs of fetal mice. The gene editors were introduced into the fetal mice 4 days prior to birth, which mimics the third trimester in humans. This 


\section{Open Access Journal of Pulmonary \& Respiratory Sciences}

approach primarily targeted pulmonary epithelial cells with alveolar type 1 , alveolar type 2 , and airway secretory cells exhibiting high and persistent gene editing. As compared to inhalation route to postnatal lungs, the intraamniotic administration gives a more uniform distribution of vector delivery.

Even though in utero delivery methods are potentially translatable, there are still many uncertainties in their application into humans. For example, the number of injections that is required to treat a human being might be too high to be practical for clinical use. In other words, intra-amniotic injection is certainly a clinically-relevant approach, but one should keep in mind that an enormous dose of vector would be required to reach anything close to the desired efficiency. Moreover, it remains unclear whether these delivery methods work effectively in humans given the huge difference in body size and the many other genetic and morphological differences between humans and rodents. Furthermore, any prenatal treatment could involve not only fetus but also the mother who is an immune competent and often diseasefree "bystander".

On the basis of their preliminary results concerning the localisation of editing system, these researchers moved to test if prenatal gene editing could rescue a clinically relevant model for a monogenic human lung disease caused by SFTPCI73Tmutation.This mutation is characterised by a substitution of threonine for isoleucine in codon 73 (I73T) and is the most common SFTPC mutation [3-5] as it is found in $>25 \%$ of patients with genetic SP-C disorders. Lung disease caused by SFTPC mutations is inherited as an autosomal dominant trait with variable penetrance and severity (45\%), or as sporadic disease caused by a de novo mutation on one allele (55\%) [5-6]. The expression of Sftpc ${ }^{173 T}$ alleles in mutant mice causes a severe diffuse parenchymal lung damage, and $100 \%$ of untreated mice will succumb to respiratory failure within hours of birth. But after treatment with in uteroCRISPR-Cas9-mediated inactivation of the mutant $\mathrm{Sftpc}^{\mathrm{I73T}}$ gene, both fetal and postnatal mice showed improvement in lung morphology and survival.

The gene editing approach used in this study presents some caveats. Indeed, these researchers applied CRISPR/Cas9/sgRNA editing system into the mouse embryo lungs with the purpose of deleting the gene, which is relatively easy, rather than repairing it, which is much harder. In other words, these researchers used CRISPR-Cas9 technology in an "alternative manner" as if
CRISPR/Cas9 editing system was a molecular scissors able to cut the mutated DNA sequence to target, without pasting a new correct DNA gene one in to replace what they have removed. Interestingly, mice born with the mutation died of lung failure within hours asSP$\mathrm{C}^{173 T}$ proprotein, which is the product of the mutated gene, is associated with a severe pathologic phenotype. In contrast, in fetuses where the gene edit succeeded (about 20 percent of them), seven survived for more than 1 day and five survived past seven days, with a restoration of lung functions. It is important to stress that, this "gene excision "was possible as Sftpc expression is not fundamental for mouse survival and lung function at normal physiologic conditions. On the contrary, SFTPC gene editing in humans, could result much more difficult as the correction of SFTPCI73T mutation would be more desirable than knocking out the endogenous gene. In other words, humans, unlike mice, need that gene, so CRISPR would have to repair it, not delete it. Moreover, mutations in SFTPC and other genes regulating surfactant production are involved in the interstitial lung disease occurring both in children and adults, accounting for just $20 \%$ of these diseases. Thus, targeting only the SFTPC gene in humans might not be enough to give rise to effective therapies by gene editing. Another important aspect is the way used to inject the CRISPR/Cas9 system into the fetus. In this study, the gene editing system has been delivered by an Ad vector, which is based on a family of viruses that cause benign respiratory tract infections in humans, are replication defective and can contain a larger amount of exogenous DNA as compared to retroviral vectors. Moreover, Ad vectors have a high transduction efficiency and a good level of gene expression although it is only transient and can deteriorate rapidly within a few weeks. However, it should be pointed out that there are also important safety concerns over the use of this type of viral vectors. Indeed, previous gene therapy studies have demonstrated that Ad vectors may cause unintended and sometimes deleterious responses from the host's immune system. In utero injection might help avoid immune sensitization against the vector system - in other words, can induce immunotolerance - as fetus is basically "immunologically naive" but much attention should be paid to the effect on the mother, which can be considered an "innocent bystander". Future works will be probably focused on investigating alternative delivery methods such as non-viral vectors or adeno-associated viruses which are less prone to stimulate unwanted immune responses in the mother. Another important consideration concerns the "time-window" in which the effects of gene editing were studied. In this study, both lung morphology and survival were evaluated at 1 week 


\section{Open Access Journal of Pulmonary \& Respiratory Sciences}

of age in Sftpc ${ }^{173 T}$ mice prenatally treated with CRISPCAs9-sgRNA.Gene editing may not provide a long-lasting response because of the natural turnover of cell populations in a given organ or tissue. This possibility may require the targeting of longer-lasting stem/progenitor cells or repeat administrations. Whether either of these approaches is more efficient or technically feasible requires further investigation.

Undoubtedly, this study will have implications for other genetic disorders where it would be safer and possibly more effective to correct a gene before fetal development is completed. Also, it constitutes a proof of concept of the chance we have to target, by in utero therapy, only affected organs as there is no need to repair the gene that cause a lung disease anywhere but lungs. Future studies should be focused on the application in larger animals to see if it is translatable, and should be oriented toward increasing the efficiency of the gene editing in this setting and evaluating different mechanisms to deliver gene editing technology to the lungs. Finally, in these experimental settings, it should be considered to create animal models that recapitulate the more complex pathogenesis of human pulmonary diseases linked to surfactant genes and to operate with the CRISPR system in amulti-modal approach. Only with these developments, the clinical application of in utero gene editing to human respiratory diseases will become a reality.

\section{References}

1. Alapati D, Zacharias WJ, Hartman HA, Rossidis AC, Stratigis JD, et al. (2019) In utero gene editing for monogenic lung disease. Science Translational Medicine 11(488).

2. Carter J, Hoffman C, Wiedenheft B (2017) The Interfaces of Genetic Conflict Are Hot Spots for Innovation. Cell 168(1-2): 9-11.

3. Percopo S, Cameron HS, Nogee LM, Pettinato G, Montella S, et al. (2004) Variable phenotype associated with SP-C gene mutations: fatal case with the 173T mutation. EurRespir J 24(6): 1072-1073.

4. Tredano M, Griese M, Brasch F, Schumacher S, de Blic J, et al. (2004) Mutation of SFTPC in infantile pulmonary alveolar proteinosis with or without fibrosing lung disease. Am J Med Genet A 126A(1): 18-26.

5. Cameron HS, Somaschini M, Carrera P, Hamvas A, Whitsett JA, et al. (2005) A common mutation in the surfactant protein $\mathrm{C}$ gene associated with lung disease. J Pediatr 146(3): 370-375.

6. Nogee LM, Dunbar AE 3rd, Wert SE, Askin F, Hamvas A, et al. (2002) Mutations in the surfactant protein C gene associated with interstitial lung disease. Chest 121(3): 20S-21S. 